\title{
Angiogenesis Inhibitors in Personalized Combination Regimens for the Treatment of Advanced Refractory Cancers
}

\section{Timothy Crook}

Broomfield Hospital

\section{Darshana Patil}

Datar Cancer Genetics (India)

\section{Rajnish Nagarkar \\ HCG Manavata Cancer Centre}

Andrew Gaya

HCA Healthcare UK

\section{Nicholas Plowman}

St Bartholomew's Hospital

\section{Sewanti Limaye}

Kokilaben Dhirubhai Ambani Hospital

\section{Navin Srivastava}

Datar Cancer Genetics (India)

\section{Vineet Datta}

Datar Cancer Genetics (India)

Dadasaheb Akolkar ( $\sim$ dadasaheb.akolkar@datarpgx.com )

Datar Cancer Genetics (India)

\section{Chirantan Bose}

Datar Cancer Genetics (India)

\section{Prashant Kumar}

Datar Cancer Genetics (India)

\section{Ajay Srinivasan}

Datar Cancer Genetics (India)

\section{Rajan Datar}

Datar Cancer Genetics (India)

\section{Research Article}

Keywords: Encyclopedic Tumor Analysis, ETA, AGI, Angiogenesis Inhibitor, Anti-Angiogenesis, VEGF, VEGFR, PDGFR, FGFR 
Posted Date: March 31st, 2021

DOI: https://doi.org/10.21203/rs.3.rs-356485/v1

License: (c) (i) This work is licensed under a Creative Commons Attribution 4.0 International License. Read Full License 


\section{Abstract \\ Background}

Angiogenic factors are commonly activated in solid tumors and present a viable therapeutic target. However, anticancer treatment with angiogenesis inhibitors (AGI) is limited to a few cancers, mostly as monotherapy and not selected based on molecular indications. We aimed to determine whether patientspecific combination regimens with $\mathrm{AGI}$ and other anticancer agents when selected based on multianalyte tumor interrogation (ETA: Encyclopedic Tumor Analysis) can expand the scope of AGIs in advanced refractory solid organ cancers with improved treatment responses.

\section{Methods}

We evaluated treatment outcomes in 60 patients with advanced, refractory solid organ cancers who received ETA-guided combination regimens of AGI with other targeted, endocrine or cytotoxic agents. Radiological evaluation of treatment response was followed by determination of Objective Response Rate (ORR), Disease Control Rate (DCR), Progression Free Survival (PFS) and Overall Survival (OS).

\section{Results}

Among the 60 patients, Partial Response (PR) was observed in 28 cases (46.7\%), Stable Disease (SD) was observed in 29 cases (48.3\%) and Disease Progression (PD, within 60 days) was observed in 3 cases (5.0\%). The ORR was $46.7 \%$ and DCR was $95.0 \%$. At the most recent follow, up the median PFS (mPFS) was 5.0 months and median OS (mOS) was 8.9 months. There were no Grade 4 therapy related adverse events or treatment related deaths.

\section{Conclusions}

ETA-guided patient-specific combination regimens with AGI and other anti-neoplastic agents, can yield improved outcomes over AGI monotherapy.

\section{Trial Registration}

Details of all trials are available at WHO-ICTRP: https://apps.who.int/trialsearch/. RESILIENT ID CTRI/2018/02/011808. LIQUID IMPACT ID CTRI/2019/02/017548.

\section{Introduction}


Tumor angiogenesis is one of the hallmarks of cancer ${ }^{1,2}$. Angiogenic factors such as VEGF ${ }^{3}, \mathrm{PDGF}^{4,5}$, $\mathrm{FGF}^{6}, \mathrm{C}^{-\mathrm{KIT}^{7}}, \mathrm{RET}^{8}, \mathrm{TIE}^{9}$ and their receptors (tyrosine kinases) such as VEGFR, PDGFR, FGFR mediate inter- and intra-cellular signalling cascades that activate cellular pathways culminating in the formation and branching of blood vessels which promotes rapid tumor growth. These angiogenesis pathways are known to cross-talk ${ }^{10}$ with other key tumor signalling pathways such as the PI3K/Akt/mTOR ${ }^{11}$, EGFR/ERBB2 ${ }^{12}$ and Raf-MEK-ERK-MAPK ${ }^{13}$ in a complex web of redundant and escape mechanisms which are favourable for tumor growth and proliferation; blockade of these signalling pathways is a viable strategy to control tumor growth and spread ${ }^{14-23}$. Several anti-angiogenic agents (angiogenesis inhibitors, AGIs) including monoclonal antibodies (mABs) such as Bevacizumab ${ }^{24}$ (anti-VEGF) or Ramucirumab $^{24}$ (anti-VEGFR2) and small molecule Tyrosine Kinase Inhibitors (TKIs) such as Axitinib ${ }^{26}$, Pazopanib ${ }^{27,28}$, Regorafenib ${ }^{29-31}$, Sorafenib ${ }^{32-34}$ and Sunitinib ${ }^{35-37}$ have been developed and approved for use in various cancers; though Imatinib ${ }^{38}$ is not a direct inhibitor of angiokinases, it's activity has been linked to downstream suppression of VEGF expression and thus angiogenesis. Unlike the mABs which are specific to a target, anti-angiogenic TKIs are known to have intracellular activity and downstream signaling a broad range of targets such as VEGFR, PDGFR, FGFR, TIE, C-KIT, RET and RAF. An overview of AGIs used in several solid organ cancers is presented in Table 1 along with molecular targets and indicated usage.

It has been shown that blockade of VEGF / VEGFR signalling axis by monotherapies often leads to transient responses followed by eventual resistance and progression ${ }^{39,40}$. Similarly, combination strategies that achieve tandem blockade of multiple signalling pathways have been proposed as a viable anticancer treatment strategy ${ }^{41-44}$. However, apart from Bevacizumab (Axitinib to a limited extent due to its use in combinations with Immune Checkpoint Inhibitors) most AGIs have been used as monotherapy and as such they offer limited therapeutic benefit. Secondly, though the molecular targets of AGIs are known, the selection of AGIs is not on the basis of tumor molecular profiling; currently there is no reliable molecular biomarker for selection of these agents. It is pertinent to add that prior efforts at informing use of targeted agents based on limited (single gene) tumor molecular profiling have yielded discouraging outcomes. Owing to these challenges, the aim of precision oncology to offer personalized (combination) regimens remains unachieved.

We have previously reported ${ }^{45,46}$ the clinical utility of multi-analyte tumor profiling (ETA: Encyclopedic Tumor Analysis) to identify latent actionable vulnerabilities in advanced refractory cancers and guide selection of safe and efficacious patient-specific combination regimens. We hypothesized that ETA can be used to inform personalized combination regimens of AGIs with other antineoplastic agents, which can yield superior or improved clinical outcomes in patients with advanced refractory solid organ cancers.

\section{Methods}

\section{Study Design and Patients}


This manuscript reports outcomes in a total of 60 adult patients who received anticancer treatment regimen informed by multi-analyte tumor profiling (ETA guided therapy). Among this cohort, 55 patients with advanced solid organ cancers, received treatments as part of either of the two ongoing prospective interventional Phase II/III trials; 38 patients in RESILIENT trial (WHO ICTRP ID CTRI/2018/02/011808) and 17 patients in LIQUID IMPACT trial (WHO ICTRP ID CTRI/2019/02/017548), into which they were enrolled between January 2018 and October 2019. All patients were previously counselled regarding study objectives, potential benefits and potential risks and provided signed written informed consent for participation in the trial and for publication of deidentified data. Both trials were approved by the ethics committees (EC) of the sponsor as well as clinical trial sites and conducted in accordance with ethical guidelines and the Declaration of Helsinki. The primary outcome data for the RESILIENT Trial has been published ${ }^{45}$ while that of the LIQUID IMPACT trial is under submission. In addition, we also report outcomes in 5 adult patients with advanced solid organ cancers who underwent ETA as a commercial service between December 2016 and June 2018 to inform precision systemic therapy options. All patients consented for analysis and publication of deidentified data. Demographic details of the Study Cohort are provided in Table 1 and Supplementary Table S01.

\section{Samples and Analysis}

All patients in the RESILIENT Trial $(n=38)$ as well as the non-trial patients $(n=5)$ provided freshly biopsied tissue samples along with peripheral blood samples obtained by venous draw. All patients in the LIQUID IMPACT Trial $(n=17)$ provided only peripheral blood samples. The collection of blood samples has been described previously ${ }^{45,46}$. ETA and generation of patient specific therapy recommendations (TR) have been described previously ${ }^{45,46}$. Briefly, ETA included (a) molecular profiling of tumor DNA, circulating tumor DNA (ctDNA), tumor RNA and exosomal RNA by Next Generation Sequencing (NGS), (b) immunocytochemistry (ICC) of Circulating Tumor Associated Cells (CTACs), and, (c) in vitro Chemoresponse Profiling (CRP) of viable tumor tissue derived cells (TDCs) or CTACs. The complete details of investigations under ETA are provided in Supplementary Methods. Supplementary Table S02 indicates the various actionable molecular indications in the Study Cohort, as informed by ETA.

\section{Treatments}

Among the 60 patients who received AGI-based regimen, 7 received additional targeted / endocrine agents, 43 received additional cytotoxic agents and 10 received additional targeted / endocrine and cytotoxic agents. A break-up of ETA-guided treatment regimen including AGIs, other Targeted and Endocrine agents and cytotoxic anticancer agents are provided in Supplementary Table S02. The selection of patient-specific combination regimens, the drug dosages and dose escalation schedules were based on the treating clinician's interpretation of ETA findings and guided by (a) institutional guidelines and protocols, (b) clinical assessment of patients' health, and (c) patient-wise list of expected treatmentrelated Adverse Events (AE)s, generated from known AE profile of single agents (from drug labels) and their combinations (drug labels and clinical trial data). Treatments were administered until disease progression or dose limiting toxicity. 


\section{Response Evaluation}

Treatment response was assessed radiologically based on a baseline and follow-up (PET-)CT scans as per RECIST 1.1 criteria $^{47}$ to determine Treatment Response as Partial Response (PR), Stable Disease (SD) or Progressive Disease (PD). Treatment response was evaluated to derive Objective Response Rate (ORR), Disease Control Rate (DCR), Progression Free Survival (PFS) and Overall Survival (OS). Trial patients underwent follow-up scans every 6-8 weeks or as advised by the treating clinician. Non-trial patients underwent follow-up scans at intervals advised by the treating clinicians.

\section{Follow-Up}

Trial patients were followed up until study termination or patient exclusion (death / loss to follow-up / withdrawal of consent) to determine Progression Free Survival (PFS) and Overall Survival (OS). Post completion of (or exclusion from) the respective study, patients were followed-up for OS.

\section{Safety and Adverse Events (AEs)}

Treatment related AEs were prospectively recorded for trial patients and retrospectively for the non-trial patients from clinical records provided by the treating clinician. All AEs were graded according to $\mathrm{NCl}-$ CTCAE $v 5^{48}$ and reported. For all patients, AEs were managed by standard procedures according to institutional protocols.

\section{Results}

\section{Molecular Profile}

The molecular landscape of angiogenesis associated genes in the study cohort is depicted in Figure 1. The most common indications were observed in PDGFR $(n=21)$, VEGFR $(n=19)$ and VEGF $(n=18)$, followed by c-KIT $(n=8)$ and FGFR $(n=5)$. Gene overexpression as determined by tumor RNA analysis was the most common variation $(n=46)$, followed by protein detection by ICC $(n=16)$. In 8 cases, mutations were observed and in 6 cases, gain of gene copy number was observed. Indications in more than 1 gene and/or more than one type of variation per gene were observed in 9 patients. Patient-wise actionable molecular features which were relevant for selection of AGIs as well as other Targeted / Endocrine anticancer agents are provided in Supplementary Table S02.

\section{Treatments}

Among the 60 patients, the multi-drug regimens included an AGI with ${ }^{3} 1$ cytotoxic agent in 43 patients and an AGI with ${ }^{3} 1$ targeted / endocrine agents in 7 patients; 10 patients received an AGI with other targeted / endocrine agents as well as ${ }^{3} 1$ cytotoxic agent(s). Only USFDA approved anticancer drugs were used in patient specific combination regimens. Selection of cytotoxic, targeted, and endocrine agents were agnostic to the respective labelled indications. Patient-wise treatment regimens including choice of 
AGIs, other Targeted and Endocrine agents and cytotoxic anticancer agents as well as the dosages are provided in Supplementary Table S02.

\section{Treatment Response}

Among the 60 patients who received ETA-guided combination regimens, there were no Complete Responses (CR), while 28 patients (46.7\%) showed Partial Response (PR), 29 patients (48.3\%) showed SD ( $\geq 60$ days) and $3(5.0 \%$ ) patients showed PD within 60 days of therapy. The Objective Response Rate (ORR) in this sub-cohort was $46.7 \%$ and Disease Control Rate (DCR) was $95.0 \%$. Patient outcomes are summarized in Table 3 and Supplementary Table S03. Among the 43 patients who received combination of AGI and cytotoxic agents (AGI_C), PR was observed in 16 patients (37.2\%). Among the remaining 17 patients where the combination regimen included an additional targeted or endocrine agent (AGI_T \pm C) PR was observed in 12 patients (70.6\%). None of the patients who received multiple targeted agents $\left(A G I \_T \pm C\right.$ ) reported PD within 60 days; all PDs All 3 patients who reported PD within 60 days were from the $A G I C C$ subgroup.

\section{Progression Free Survival (PFS) and Overall Survival (OS)}

Among the 60 patients, median Progression Free Survival and Median Overall Survival (mPFS and m0S, respectively) were 5.0 months ( $95 \% \mathrm{Cl}: 4.1$ - 5.8 months) and 8.9 months ( $95 \% \mathrm{Cl}: 7.3-10.6$ months) respectively. Among the 43 patients in the AGI_C subgroup, mPFS and mOS were 4.4 months and 8.8 months respectively, whereas in the $A G I_{-} T \pm C$ subgroup, these values were incrementally higher 6.7 and 10.0 months, respectively (the cohort sizes do not permit calculation of statistical significance). Kaplan Meier Plots of PFS and OS are presented in Figure 2.

We benchmarked the PFS of each patient on ETA-guided combination regimen (PFS2) against PFS on patient's last failed line of therapy (PFS1) (Figure 3). With a median PFS1 of 3.1 (95\%Cl: $1.1-5.1$ months) months, the overall PFS2:PFS1 ratio of 1.6, indicating therapeutic benefit leading to significant increase in PFS. The PFS2:PFS1 ratios were $\geq 2.50$ in 16 patients (26.7\%), , $1.31-1.49$ in 19 patients (31.7\%), and , $0.81-1.29$ in 13 patients (21.7\%). Among the 12 patients with PFS2:PFS1 ratio of $\leq 0.8$ were 6 patients with recurrent disease (>12 months gap). Patient outcomes are summarized in Table 3 and Supplementary Table S03.

\section{Treatment Related Adverse Events}

Grade I / II Treatment-Related Adverse Events (AE) were observed in all 60 patients (Table 4). Treatment related Grade III AEs were observed in 45 patients. Grade IV treatment related AEs were not reported, nor were any treatment related deaths. The most common Grade I /II AEs included Fatigue, Anorexia, Mucositis, Nausea, which also were reported as Grade III AEs albeit at lower frequencies. There were no significant differences between profile of $A E s$ depending on the nature of the treatment regimen (i.e., AGI_C v/s AGI_T \pm C). Patient-wise AEs are provided in Supplementary Table S04. 


\section{Discussion}

The findings of the present study show that co-administration of AGI and other anticancer agents are well tolerated and achieve significant response even in a heavily pre-treated cohort with advanced solid organ cancers. It is generally accepted that the probability of success of anticancer treatments decrease with successive lines of treatment. However, our study shows a significant increase in median PFS as compared to the patients' last failed line of treatment. Since several patients are continuing to receive treatments at the time of submission, we anticipate further improvements to the treatment benefit metrics reported in this manuscript.

Presently, the selection of AGI for use in anticancer treatment regimens is not informed by molecular genetic indication. This is the case for most, if not all, targeted anticancer agents such as tyrosine kinase inhibitors (TKI) as well as mABs. Though the paradigm of personalized label agnostic treatment selection based on tumor molecular profiling has been explored for several targeted anticancer agents, this appears to be less so in case of AGIs where there are fewer studies on organ agnostic treatment selection. Among the NCI-MATCH ${ }^{49}$ basket trials, there are 3 trials for FGFR aberrations and 1 trial on cKIT aberrations for selection of AGl; interim results for one of these studies indicated absence of significant treatment benefit with $9 \% \mathrm{ORR}^{50}$. Most AGIs are administered as monotherapy which yield modest treatment benefits Prior studies have established the benefits of combination regimens of various TKIs in comparison to monotherapy with the same agents. In several cancers, targeted agents are administered with other targeted or endocrine or cytotoxic agents; Axitinib and Bevacizumab appear to be researched more extensively in comparison to other AGIs reported in this manuscript. Several combinations of Axitinib and Bevacizumab with other targeted agents, checkpoint inhibitors or cytotoxic agents are presently approved for treatment of various cancers since they offer varying levels of improvement over existing monotherapy options. In a recent study ${ }^{51}$ it was observed that VEGF blockade, though effective in suppressing the primary tumour, could present hypoxic conditions conducive to release and survival of CTC clusters with metastatic potential. It follows that a combination regimen that targets angiogenesis as well as other activated pathways or cellular mechanisms (such as e.g., DNA replication, DNA synthesis, or mitosis) in tandem could avoid such potential pitfalls by acting on CTCs or clusters released from the tumor and are no longer susceptible to inhibition of angiogenesis.

The present study was designed to select AGIs based on molecular indications for use in patient specific combination regimens with other anticancer drugs which were selected based on de novo functional and molecular profiling of the tumors. The scope of AGIs in the present study was limited by the availability of approved drugs at the trial location and included Axitinib, Bevacizumab, Pazopanib, Regorafenib, Sorafenib and Sunitinib; Imatinib, though not a classical AGI is known to inhibit angiogenesis as a downstream function and was also considered. Except for Bevacizumab, a mAb specific for VEGF, all other anticancer agents are small molecule TKls with varying activities against a repertoire of angiogenesis-related factors including VEGFR, PDGFR, FGFR, c-KIT, RET, RET families. Accordingly, ETA evaluated gene alterations including single nucleotide variations (SNV) and copy number alterations (CNA, specifically gain of copy number) as well as overexpression of all genes where the polypeptide 
product can be targeted by AGIs. While SNV and CNA were determined by NGS, gene expression was evaluated by NGS of tumor tissue RNA or exosomal RNA, as well as by ICC of peripheral blood C-TACs. The study cohort included 18 patients who were unable to undergo a biopsy for tumor profiling - in these patients, peripheral blood tumor analytes including circulating tumor DNA (ctDNA), exosomal RNA and CTACs were evaluated.

The molecular profiles of angiogenesis related genes in each patient are illustrated in Fig 1. In 21 patients, molecular features associated with multiple angiogenesis related genes was observed while in 3 patients, multiple alterations in same gene (copy number variation as well as point mutation) were observed. This aided the selection of AGIs that can efficiently target multiple vulnerabilities. In 17 patients, ETA also identified other tumor features such as (but not limited to) activation of mTOR or hormone receptor signalling pathways. In these patients, the corresponding targeted agent or endocrine antagonist was evaluated for incorporation in the treatment regimen.

The study findings suggest that ETA based comprehensive multi-analyte cancer profiling provides relevant molecular and functional evidence which in turn can inform selection of patient-specific combination regimens of AGIs with other anticancer agents. The benefits of such combination regimens may exceed those obtained with arbitrary selection of monotherapy AGIs or their combination regimens, respectively. While the merits of combination treatment strategies have been expounded previously ${ }^{52-54}$, the safety of such regimens are an important consideration. Meta-analyses of clinical trials have observed that de novo combinations of targeted and cytotoxic agents can be safely administered to patients without any increased risks of toxicity. The present study comprised exclusively of heavily pretreated cases of advanced cancers with an inherently higher risk of AEs due to cumulative toxicities from prior treatments; ETA guided combination regimens of AGI and other anticancer agents were generally well tolerated with manageable profile of treatment related AEs. Though the present study did not include any therapy naïve patients, we speculate that ETA-guided approach can be beneficial as first line for advanced metastatic cases or in the neoadjuvant setting for other (operable) cases. In conclusion, our study demonstrates (a) the clinical efficacy of combination regimens with AGI and other anticancer agents in yielding superior response rates and survival benefits, and (b) the ability of ETA to guide selection of safe and efficacious treatment strategies in heavily pretreated patients with advanced refractory cancers.

\section{Abbreviations}

AGI: Angiogenesis Inhibitor; ETA: Encyclopedic Tumor Analysis; ORR: Objective Response Rate; DCR: Disease Control Rate; mPFS: median Progression Free Survival; mOS: median Overall Survival; VEGF: Vascular Endothelial Growth Factor; VEGFR: Vascular Endothelial Growth Factor Receptor; FGF: Fibroblast Growth Factor; FGFR: Fibroblast Growth Factor Receptor; PDGF: Platelet Derived Growth Factor; PDGFR: Platelet Derived Growth Factor Receptor; c-KIT: Cellular Homolog of the Feline Sarcoma Viral Oncogene v-KIT; RAF: Rapidly Accelerated Fibrosarcoma (protein kinase); RET: Rearranged during Transfection (protein kinase); BCR-ABL: fusion of ABL1 and BCR gene products (protein kinase); RCT: 
Randomised Clinical Trials; SoC: Standard of Care; ARC: advanced refractory cancers; IHC: Immunohistochemistry; ICC: Immunocytochemistry; USFDA: United States Food and Drug Administration; CR: Complete Response; PR: Partial Response; SD: Stable Disease; PD: Progressive Disease (Disease Progression); PFS: Progression Free Survival; OS: Overall Survival; AE: Adverse Event; ctDNA: Circulating (cell-free) tumor DNA; SNV: Single Nucleotide Variations; CNV: Copy Number Variations; ReSP: Resistance and Sensitivity Profiling; NGS: Next Generation Sequencing; DGE: Differential Gene Expression; TDCs: Tumor Tissue Derived Cells; C-TACs: Circulating Tumor Associated Cells; RECIST: Response Evaluation Criteria in Solid Tumors; CTCAE: Common Terminology Criteria for Adverse Events;

\section{Declarations}

\section{Ethics Approval and Consent to Participate}

All trials were approved by institutional review boards and ethics committees of the study sponsor (Datar Cancer Genetics, DCG) as well as clinical trial sites (HCG Manavata Cancer Centre, HCG-MCC). All trials were conducted in accordance with all applicable ethical guidelines and the Declaration of Helsinki. All study participants were previously counselled regarding study objectives, potential benefits and potential risks and provided signed written informed consent for participation in the trial.

\section{Consent for Publication}

All study participants consented for publication of deidentified biological data. The present manuscript does not contain any personal or identifiable information or data of any participant. All Authors have consented to publication of this manuscript and the data.

\section{Data Availability}

The datasets generated during and/or analysed during the current study are available from the corresponding author on reasonable request.

\section{Competing Interests}

TC, RN, AG, NP and SL have no competing interests; DP, NS, VD, CB, PK, DA and AS are employees of the Study Sponsor; RD is the founder of the Study Sponsor

\section{Funding}

No external funding was obtained for this study. The entire study was funded by the Study Sponsor (DCG).

\section{Authors' contributions}

TC: Study Design, Data Review, Manuscript Review; 
DP: Study Design, Data Review, Data Analysis, Manuscript Review;

RN: Study Design, Clinical Management, Manuscript Review;

AG: Study Design, Data Review, Manuscript Review;

NP: Study Design, Data Review, Manuscript Review;

SL: Study Design, Clinical Management, Manuscript Review;

NS: Study Design, Molecular Analysis, Data Analysis, Manuscript Review;

VD: Study Design, Data Review, Manuscript Review;

CB: Data Review, Data Analysis, Manuscript Review;

PK: Data Review, Manuscript Review;

DA: Study Design, Molecular Analysis, Data Review, Manuscript Review;

AS: Study Design, Data Analysis, Manuscript Writing;

RD: Resources, Study Design, Manuscript Review.

\section{ACKNOWLEDGEMENTS}

The authors are grateful towards all study participants and their caregivers. The contributions of HCG Manavata Cancer Centre, Avinash Cancer Centre, as well as the staff of the Study Sponsor (DCG) towards managing various clinical, operational and laboratory aspects of the study are also acknowledged with gratitude.

\section{References}

1. El-Kenawi $A E$, El-Remessy $A B$. Angiogenesis inhibitors in cancer therapy: mechanistic perspective on classification and treatment rationales. Br J Pharmacol. 2013;170(4):712-29. doi:10.1111/bph.12344.

2. Cook KM, Figg WD. Angiogenesis inhibitors: current strategies and future prospects. CA Cancer J Clin. 2010;60(4):222-43. doi:10.3322/caac.20075.

3. Hicklin DJ, Ellis LM. Role of the vascular endothelial growth factor pathway in tumor growth and angiogenesis. J Clin Oncol. 2005;23(5):1011-27. doi:10.1200/JC0.2005.06.081.

4. Farooqi AA, Siddik ZH. Platelet-derived growth factor (PDGF) signalling in cancer: rapidly emerging signalling landscape. Cell Biochem Funct. 2015;33(5):257-65. doi:10.1002/cbf.3120.

5. Gialeli C, Nikitovic D, Kletsas D, Theocharis AD, Tzanakakis GN, Karamanos NK. PDGF/PDGFR signaling and targeting in cancer growth and progression: Focus on tumor microenvironment and 
cancer-associated fibroblasts. Curr Pharm Des. 2014;20(17):2843-8.

doi:10.2174/13816128113199990592.

6. Korc M, Friesel RE. The role of fibroblast growth factors in tumor growth. Curr Cancer Drug Targets. 2009;9(5):639-51. doi:10.2174/156800909789057006.

7. Foster BM, Zaidi D, Young TR, Mobley ME, Kerr BA. CD117/c-kit in Cancer Stem Cell-Mediated Progression and Therapeutic Resistance. Biomedicines. 2018;6(1):31. Published 2018 Mar 8. doi:10.3390/biomedicines6010031.

8. Jhiang SM. The RET proto-oncogene in human cancers. Oncogene. 2000;19(49):5590-7. doi:10.1038/sj.onc.1203857.

9. Partanen J, Dumont DJ. Functions of Tie1 and Tie2 receptor tyrosine kinases in vascular development. Curr Top Microbiol Immunol. 1999;237:159-72. doi:10.1007/978-3-642-59953-8_8.

10. Lai Y, Zhao Z, Zeng T, et al. Crosstalk between VEGFR and other receptor tyrosine kinases for TKI therapy of metastatic renal cell carcinoma. Cancer Cell Int. 2018;18:31. https://doi.org/10.1186/s12935-018-0530-2.

11. Conciatori F, Ciuffreda L, Bazzichetto C, et al. mTOR Cross-Talk in Cancer and Potential for Combination Therapy. Cancers (Basel). 2018;10(1):23. Published 2018 Jan 19. doi:10.3390/cancers10010023.

12. van Cruijsen H, Giaccone G, Hoekman K. Epidermal growth factor receptor and angiogenesis: Opportunities for combined anticancer strategies. Int J Cancer. 2005;117(6):883-8. doi:10.1002/ijc.21479.

13. Song M, Finley SD. Mechanistic insight into activation of MAPK signaling by pro-angiogenic factors. BMC Syst Biol. 2018;12:145. https://doi.org/10.1186/s12918-018-0668-5.

14. Shibuya M. Vascular Endothelial Growth Factor (VEGF) and Its Receptor (VEGFR) Signaling in Angiogenesis: A Crucial Target for Anti- and Pro-Angiogenic Therapies. Genes Cancer. 2011;2(12):1097-105. doi:10.1177/1947601911423031.

15. Sitohy B, Nagy JA, Dvorak HF. Anti-VEGF/VEGFR therapy for cancer: reassessing the target. Cancer Res. 2012;72(8):1909-14. doi:10.1158/0008-5472.CAN-11-3406.

16. Navid S, Fan C, Flores-Villanueva O, Generali P, Li D. Y. The Fibroblast Growth Factor Receptors in Breast Cancer: from Oncogenesis to Better Treatments. Int J Mol Sci. 2020;21(6):2011. doi:10.3390/ijms21062011. Published 2020 Mar 16.

17. Dieci MV, Arnedos M, Andre F, Soria JC. Fibroblast growth factor receptor inhibitors as a cancer treatment: from a biologic rationale to medical perspectives. Cancer Discov. 2013;3(3):264-79. doi:10.1158/2159-8290.CD-12-0362.

18. Heldin $\mathrm{CH}$. Targeting the PDGF signaling pathway in tumor treatment. Cell Commun Signal. 2013;11:97. doi:10.1186/1478-811X-11-97. Published 2013 Dec 20.

19. Pietras K, Sjöblom T, Rubin K, Heldin $\mathrm{CH}$, Ostman A. PDGF receptors as cancer drug targets. Cancer Cell. 2003;3(5):439-43. doi:10.1016/s1535-6108(03)00089-8. 
20. Abbaspour Babaei M, Kamalidehghan B, Saleem M, Huri HZ, Ahmadipour F. Receptor tyrosine kinase (c-Kit) inhibitors: a potential therapeutic target in cancer cells. Drug Des Devel Ther. 2016;10:244359. doi:10.2147/DDDT.S89114. Published 2016 Aug 1.

21. Stankov K, Popovic S, Mikov M. C-KIT signaling in cancer treatment. Curr Pharm Des. 2014;20(17):2849-80. doi:10.2174/13816128113199990593.

22. Subbiah V, Cote GJ. Advances in Targeting RET-Dependent Cancers. Cancer Discov. 2020;10(4):498505. doi:10.1158/2159-8290.CD-19-1116.

23. Press MF, Lenz HJ. EGFR, HER2 and VEGF pathways: validated targets for cancer treatment. Drugs. 2007;67(14):2045-75. doi:10.2165/00003495-200767140-00006.

24. Shih T, Lindley C. Bevacizumab: an angiogenesis inhibitor for the treatment of solid malignancies. Clin Ther. 2006;28(11):1779-802. doi:10.1016/j.clinthera.2006.11.015.

25. Fala L. Cyramza (Ramucirumab) Approved for the Treatment of Advanced Gastric Cancer and Metastatic Non-Small-Cell Lung Cancer. Am Health Drug Benefits. 2015;8(Spec Feature):49-53.

26. Rini BI, Escudier B, Tomczak P, et al. Comparative effectiveness of axitinib versus sorafenib in advanced renal cell carcinoma (AXIS): a randomised phase 3 trial [published correction appears in Lancet. 2012 Nov 24;380(9856):1818]. Lancet. 2011;378(9807):1931-1939. doi:10.1016/S01406736(11)61613-9.

27. Sternberg CN, Davis ID, Mardiak J, et al. Pazopanib in locally advanced or metastatic renal cell carcinoma: results of a randomized phase III trial. J Clin Oncol. 2010;28(6):1061-8. doi:10.1200/JC0.2009.23.9764.

28. van der Graaf WT, Blay JY, Chawla SP, et al. Pazopanib for metastatic soft-tissue sarcoma (PALETTE): a randomised, double-blind, placebo-controlled phase 3 trial. Lancet. 2012;379(9829):1879-86. doi:10.1016/S0140-6736(12)60651-5.

29. Grothey A, Van Cutsem E, Sobrero A, et al. Regorafenib monotherapy for previously treated metastatic colorectal cancer (CORRECT): an international, multicentre, randomised, placebocontrolled, phase 3 trial. Lancet. 2013;381(9863):303-12. doi:10.1016/S0140-6736(12)61900-X.

30. Demetri GD, Reichardt P, Kang YK, et al. Efficacy and safety of regorafenib for advanced gastrointestinal stromal tumours after failure of imatinib and sunitinib (GRID): an international, multicentre, randomised, placebo-controlled, phase 3 trial. Lancet. 2013;381(9863):295-302. doi:10.1016/S0140-6736(12)61857-1.

31. Bruix J, Qin S, Merle P, et al. Regorafenib for patients with hepatocellular carcinoma who progressed on sorafenib treatment (RESORCE): a randomised, double-blind, placebo-controlled, phase 3 trial [published correction appears in Lancet. 2017 Jan 7;389(10064):36]. Lancet. 2017;389(10064):5666. doi:10.1016/S0140-6736(16)32453-9.

32. Llovet JM, Ricci S, Mazzaferro V, et al. Sorafenib in advanced hepatocellular carcinoma. N Engl J Med. 2008;359(4):378-90. doi:10.1056/NEJMoa0708857.

33. Wilhelm SM, Carter C, Tang L, et al. BAY 43-9006 exhibits broad spectrum oral antitumor activity and targets the RAF/MEK/ERK pathway and receptor tyrosine kinases involved in tumor progression and 
angiogenesis. Cancer Res. 2004;64(19):7099-109. doi:10.1158/0008-5472.CAN-04-1443.

34. Brose MS, Nutting CM, Jarzab B, et al. Sorafenib in radioactive iodine-refractory, locally advanced or metastatic differentiated thyroid cancer: a randomised, double-blind, phase 3 trial. Lancet. 2014;384(9940):319-28. doi:10.1016/S0140-6736(14)60421-9.

35. Demetri DG, van Oosterom A, Blackstein $\mathrm{M}$, et al. Phase 3 multicenter, randomized, double-bind, placebo-controlled trial of SU11248 in patients following failure of imatinib for metastatic GIST. American Society of Clinical Oncology 41st Annual Meeting; 13-17 May; Orlando, Florida, USA. 2005 DOI: 10.1200/jco.2005.23.16_suppl.4000.

36. Ravaud A, Motzer RJ, Pandha HS, et al. Adjuvant Sunitinib in High-Risk Renal-Cell Carcinoma after Nephrectomy. N Engl J Med. 2016;375(23):2246-54. doi:10.1056/NEJMoa1611406.

37. Raymond E, Dahan L, Raoul JL, et al. Sunitinib malate for the treatment of pancreatic neuroendocrine tumors [published correction appears in N Engl J Med. 2011 Mar 17;364(11):1082]. N Engl J Med. 2011;364(6):501-513. doi:10.1056/NEJMoa1003825.

38. Dagher R, Cohen M, Williams G, et al. Approval summary: imatinib mesylate in the treatment of metastatic and/or unresectable malignant gastrointestinal stromal tumors. Clin Cancer Res. 2002;8(10):3034-8.

39. Zhao Y, Adjei AA. Targeting Angiogenesis in Cancer Therapy: Moving Beyond Vascular Endothelial Growth Factor. Oncologist. 2015;20(6):660-73. doi:10.1634/theoncologist.2014-0465.

40. Haibe $Y$, Kreidieh M, El Hajj $H$, et al. Resistance Mechanisms to Anti-angiogenic Therapies in Cancer. Front Oncol. 2020;10:221. doi:10.3389/fonc.2020.00221. Published 2020 Feb 27.

41. Deng H, Kan A, Lyu N, et al. Dual Vascular Endothelial Growth Factor Receptor and Fibroblast Growth Factor Receptor Inhibition Elicits Antitumor Immunity and Enhances Programmed Cell Death-1 Checkpoint Blockade in Hepatocellular Carcinoma. Liver Cancer. 2020;9(3):338-57. doi:10.1159/000505695.

42. Qin S, Li A, Yi M, Yu S, Zhang M, Wu K. Recent advances on anti-angiogenesis receptor tyrosine kinase inhibitors in cancer therapy. J Hematol Oncol. 2019;12(1):27. Published 2019 Mar 12. doi:10.1186/s13045-019-0718-5.

43. Gotink KJ, Verheul HM. Anti-angiogenic tyrosine kinase inhibitors: what is their mechanism of action? Angiogenesis. 2010;13(1):1-14. doi:10.1007/s10456-009-9160-6.

44. Yi M, Jiao D, Qin S, et al. Synergistic effect of immune checkpoint blockade and anti-angiogenesis in cancer treatment. Mol Cancer. 2019;18:60. ttps://doi.org/10.1186/s12943-019-0974-6.

45. Ranade A, Patil D, Bhatt A, et al. Adaptive, Iterative, Long-Term Personalized Therapy Management in a Case of Stage IV Refractory NSCLC. J Pers Med. 2019 Jul;5(3):34. 9(.

46. Nagarkar R, Patil D, Crook T, et al. Encyclopedic tumor analysis for guiding treatment of advanced, broadly refractory cancers: results from the RESILIENT trial. Oncotarget. 2019;10(54):5605-21. doi:10.18632/oncotarget.27188. Published 2019 Sep 24.

47. Eisenhauer EA, Therasse P, Bogaerts $\mathrm{J}$, et al. New response evaluation criteria in solid tumours: revised RECIST guideline (version 1.1). Eur J Cancer. 2009;45(2):228-47. 
48. NCI. NIH, DHHS. Common Terminology Criteria for Adverse Events v5.0. 2017.

49. NCI-MATCH. https://ecog-acrin.org/trials/nci-match-eay131. Accessed on 20-Aug-2020.

50. Chae YK, Hong F, Vaklavas C, et al. Phase II Study of AZD4547 in Patients With Tumors Harboring Aberrations in the FGFR Pathway: Results From the NCI-MATCH Trial (EAY131) Subprotocol W. J Clin Oncol. 2020;38(21):2407-17. doi:10.1200/JC0.19.02630.

51. Donato C, Kunz L, Castro-Giner F, et al. Hypoxia Triggers the Intravasation of Clustered Circulating Tumor Cells. Cell Rep. 2020 Sep 8;32(10):108105.

52. Liu S, Nikanjam M, Kurzrock R. Dosing de novo combinations of two targeted drugs: Towards a customized precision medicine approach to advanced cancers. Oncotarget. 2016;7:11310-20.

53. Nikanjam M, Liu S, Yang J, Kurzrock R. Dosing Three-Drug Combinations That Include Targeted AntiCancer Agents: Analysis of 37,763 Patients. Oncologist. 2017 May;22(5):576-84.

54. Nikanjam M, Liu S, Kurzrock R. Dosing targeted and cytotoxic two-drug combinations: Lessons learned from analysis of 24,326 patients reported 2010 through 2013. Int J Cancer. 2016 Nov $1 ; 139(9): 2135-41$.

\section{Tables}

Table 1

Details of Angiogenesis Inhibitors (AGIs) administered in the present study, their targets used in epithelial and solid organ cancers. Apart from Bevacizumab which is a monoclonal antibody (mAb) specific for VEGF, all other AGIs are small molecular tyrosine kinase inhibitors (TKIs) which can inhibit multiple targets. Combinations of Bevacizumab are frequently approved in several cancers. Apart from Axitinib, other TKIs are largely used as monotherapy. None of the AGIs are selected on the basis of tumor profiling for these targets.

\begin{tabular}{|c|c|c|}
\hline Drug & Target(s) & Use in Cancers \\
\hline Bevacizumab & VEGF & GBM; $\mathrm{CRC}^{1} ; \mathrm{NSCLC}^{2}$; Cervix ${ }^{3} ;$ Ovary $^{4} ; \mathrm{RCC}^{5} ; \mathrm{HCC}^{6}$ \\
\hline Axitinib & VEGFR, PDGFR, c-KIT & $\mathrm{RCC}^{7}$ \\
\hline Imatinib & BCR-ABL, PDGFR, c-KIT & GIST \\
\hline Pazopanib & VEGFR, PDGFR, c-KIT, FGFR & RCC, Sarcomas \\
\hline Regorafenib & VEGFR, PDGFR, c-KIT, RET & CRC, GIST, HCC \\
\hline Sorafenib & VEGFR, PDGFR & RCC, HCC, Thyroid \\
\hline Sunitinib & VEGFR, PDGFR, c-KIT, RET & GIST, Pancreatic NET, RCC \\
\hline
\end{tabular}

GBM: Glioblastoma; CRC: Colorectal Cancer, NSCLC: Non-Small Cell Lung Cancer; RCC: Renal Cell Carcinoma; HCC: Hepatocellular Carcinoma; GIST: Gastrointestinal Stromal Tumor; NET: Neuroendocrine Tumor. ${ }^{1}$ with 
FOLFOX / FOLFIRI / FOLFIRINOX; ${ }^{2}$ with Carboplatin and Paclitaxel; ${ }^{3}$ with Paclitaxel and Cisplatin / Topotecan;

${ }^{4}$ with Paclitaxel and Carboplatin; ${ }^{5}$ with IFN- $\alpha$; ${ }^{6}$ with Atezolizumab; ${ }^{7}$ as monotherapy as well as with Pembrolizumab / Avelumab.

Table 2

Study Cohort. The present study reports outcomes in a curated cohort of 60 adult patients with advanced refractory solid organ cancers.

\begin{tabular}{|ll|}
\hline Parameter & Value \\
\hline Gender & 30 \\
Female & 30 \\
Male & \\
\hline Age & $49(22-71)$ \\
Median (Range) & \\
Cancer Type & 11 \\
Breast (IDC) & 1 \\
Cervix (NET) & 5 \\
Colorectum (AD) & 1 \\
Duodenum (NET) & 2 \\
Esophagus (SCC) & 13 \\
Head and Neck & 3 \\
(SCC) & 2 \\
Kidney (RCC) & 1 \\
Liver (HCC) & 1 \\
Lung (AD) & 1 \\
Lung (SCC) & 2 \\
Melanoma & 7 \\
Occult (NET) & 3 \\
Ovary (AD) & 1 \\
Pancreas (AD) & 1 \\
Pilomatrixoma & 4 \\
Sarcoma & 1 \\
Stomach (AD) & \\
Testes (NSCGT) & \\
\hline
\end{tabular}

IDC: Invasive / Infiltrating Ductal Carcinoma; SCC: Squamous Cell Carcinoma; AD: Adenocarcinoma; NET: Neuroendocrine Tumor; RCC: Renal Cell Carcinoma; HCC: Hepatocellular Carcinoma; PMX: Pilomatrixoma; NSGCT: Non-Seminomatous Germ Cell Tumor. 
Table 3

Outcomes of ETA-guided Combination Regimens of AGI and other Targeted, Endocrine and Cytotoxic agents.

\begin{tabular}{|llll|}
\hline Parameter & Overall & AGICC & AGI_T \pm C \\
\hline Patients & 60 & 43 & 17 \\
\hline Outcome & $28(46.7 \%)$ & $16(37.2 \%)$ & $12(70.6 \%)$ \\
PR & $29(48.3 \%)$ & $24(55.8 \%)$ & $5(29.4 \%)$ \\
SD & $3(5.0 \%)$ & $3(7.0 \%)$ & \\
PD & & & \\
Response & $46.7 \%$ & $37.2 \%$ & $70.6 \%$ \\
Rates & $95.0 \%$ & $93.0 \%$ & $100.0 \%$ \\
ORR & & & \\
DCR & & & \\
Survival* & $5.0(4.1-$ & $4.4(3.4-$ & $6.7(4.8-8.5)$ \\
mPFS & $5.8)$ & $5.3)$ & $10.0(7.2-12.9)$ \\
mOS & $8.9(7.3-$ & $8.8(6.7-$ & \\
& $10.6)$ & $10.9)$ & \\
\hline
\end{tabular}

IDC: Invasive / Infiltrating Ductal Carcinoma; SCC: Squamous Cell Carcinoma; AD: Adenocarcinoma; NET: Neuroendocrine Tumor; RCC: Renal Cell Carcinoma; HCC: Hepatocellular Carcinoma; PMX: Pilomatrixoma; NSGCT: Non-Seminomatous Germ Cell Tumor. 
Table 4

Profile of Known and Likely Treatment Related Adverse Events. There were no recorded Grade IV Treatment Related AEs nor any Treatment Related Deaths. Patient-wise AEs reported as well as AEs in treatment subgroups (AGI_C, AGI_T \pm C) are provided in Supplementary Table S04.

\begin{tabular}{|lllll|}
\hline Adverse Event & \multicolumn{3}{l}{ Grade I II } & \multicolumn{2}{l|}{ Grade III } \\
\hline Anemia & 9 & $15.0 \%$ & 4 & $6.7 \%$ \\
\hline Anorexia & 41 & $68.3 \%$ & 9 & $15.0 \%$ \\
\hline Constipation & 9 & $15.0 \%$ & 2 & $3.3 \%$ \\
\hline Diarrhoea & 13 & $21.7 \%$ & 2 & $3.3 \%$ \\
\hline Edema & 11 & $18.3 \%$ & 3 & $5.0 \%$ \\
\hline Fatigue & 54 & $90.0 \%$ & 28 & $46.7 \%$ \\
\hline Hyper-/Hypotension & 5 & $8.3 \%$ & 3 & $5.0 \%$ \\
\hline Mucositis Oral & 27 & $45.0 \%$ & 9 & $15.0 \%$ \\
\hline Myalgia & 14 & $23.3 \%$ & 4 & $6.7 \%$ \\
\hline Nausea & 21 & $35.0 \%$ & 4 & $6.7 \%$ \\
\hline Neutropenia & 17 & $28.3 \%$ & 5 & $8.3 \%$ \\
\hline Peripheral & 6 & $10.0 \%$ & 2 & $3.3 \%$ \\
\hline Neuropathy & & & & $3.3 \%$ \\
\hline Rash / Itch & 10 & $16.7 \%$ & 2 & $3.3 \%$ \\
\hline Pyrexia & 15 & $25.0 \%$ & 2 & $16.7 \%$ \\
\hline Thrombocytopenia & 12 & $20.0 \%$ & 10 & $1.7 \%$ \\
\hline Vomiting & 11 & $18.3 \%$ & 1 & \\
\hline
\end{tabular}




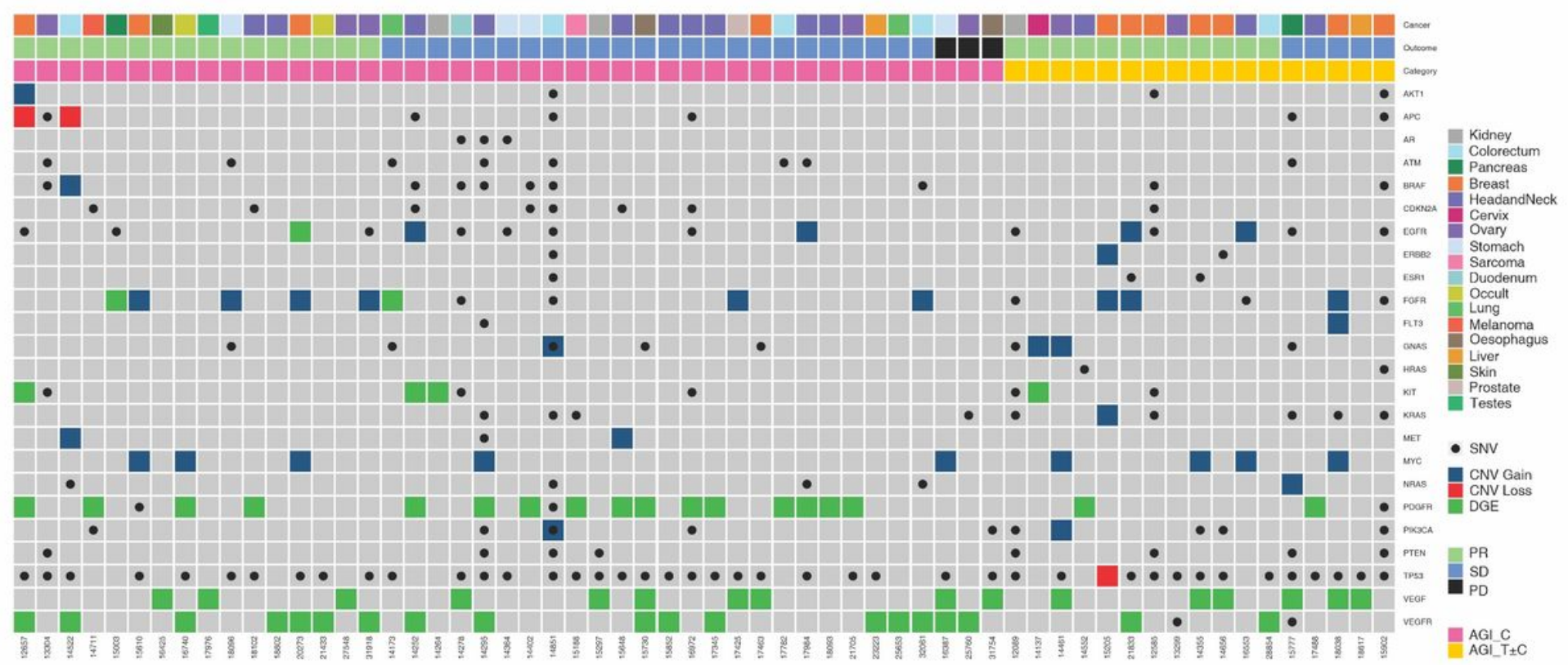

\section{Figure 1}

Molecular Landscape of Angiogenesis Factors and their Receptors in the Study Cohort. Molecular features associated with angiogenesis factor and receptor genes are depicted. Five-digit numbers at the bottom of each column indicate individual patients in the study cohort. Cancer types (topmost row) and gender (second row from top) are colour coded. SNV: Single Nucleotide Variation; CNV: Copy Number Variation; PR: Partial Response; SD: Stable Disease; PD: Disease Progression.
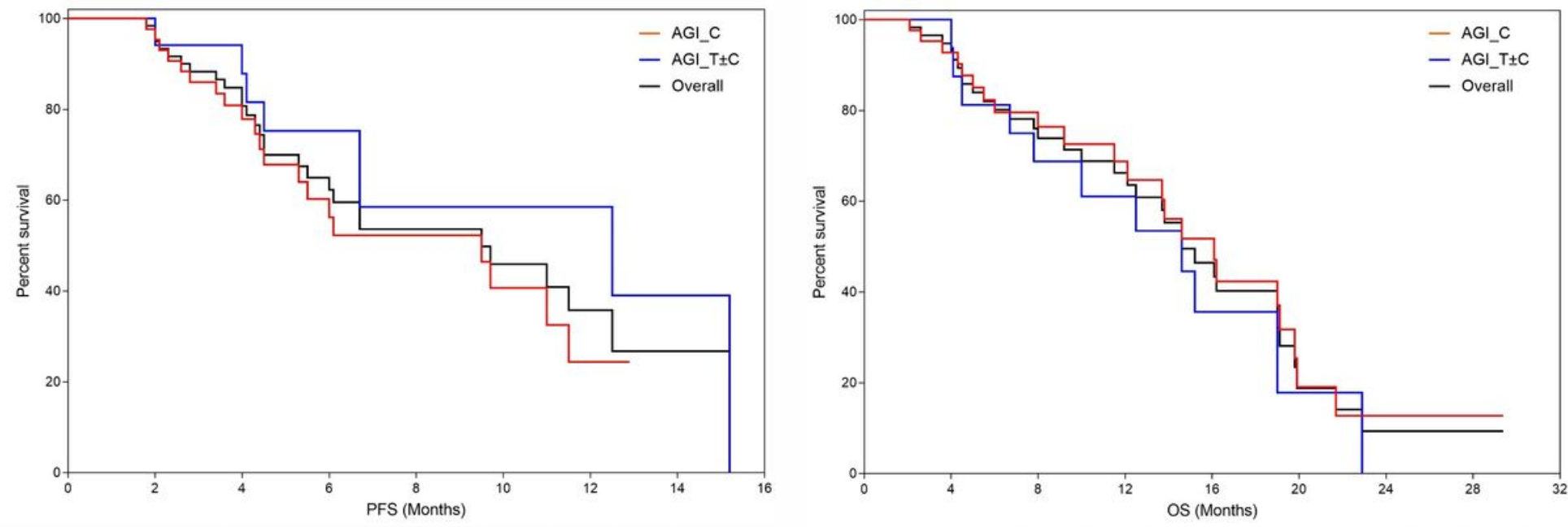

\begin{tabular}{|c|c|c|c|c|c|c|c|c|}
\hline At Risk & $\mathbf{0}$ & $\mathbf{2}$ & $\mathbf{4}$ & $\mathbf{6}$ & $\mathbf{8}$ & $\mathbf{1 0}$ & $\mathbf{1 2}$ & $\mathbf{1 4}$ \\
\hline AGI_C & 43 & 42 & 27 & 15 & 10 & 8 & 3 & 1 \\
\hline AGI_T $\pm C$ & 17 & 17 & 15 & 9 & 7 & 5 & 3 & 2 \\
\hline Overall & 60 & 59 & 42 & 24 & 17 & 13 & 6 & 3 \\
\hline
\end{tabular}

\begin{tabular}{|c|c|c|c|c|c|c|c|c|}
\hline At Risk & $\mathbf{0}$ & $\mathbf{4}$ & $\mathbf{8}$ & $\mathbf{1 2}$ & $\mathbf{1 6}$ & $\mathbf{2 0}$ & $\mathbf{2 4}$ & $\mathbf{2 8}$ \\
\hline AGI_C & 43 & 37 & 25 & 19 & 11 & 4 & 2 & 2 \\
\hline AGI_T士C & 17 & 16 & 11 & 8 & 5 & 1 & 1 & 0 \\
\hline Overall & 60 & 53 & 36 & 27 & 16 & 5 & 3 & 2 \\
\hline
\end{tabular}

\section{Figure 2}

Kaplan Meier Plots of Progression Free Survival and Overall Survival. Progression Free Survival (PFS, A) and Overall Survival (OS, B) were evaluated based on regimen subtypes: AGI in combination with Cytotoxic Agents (AGI_C) or other targeted agents irrespective of cytotoxic agents (ETA_T $\pm C$ ). 


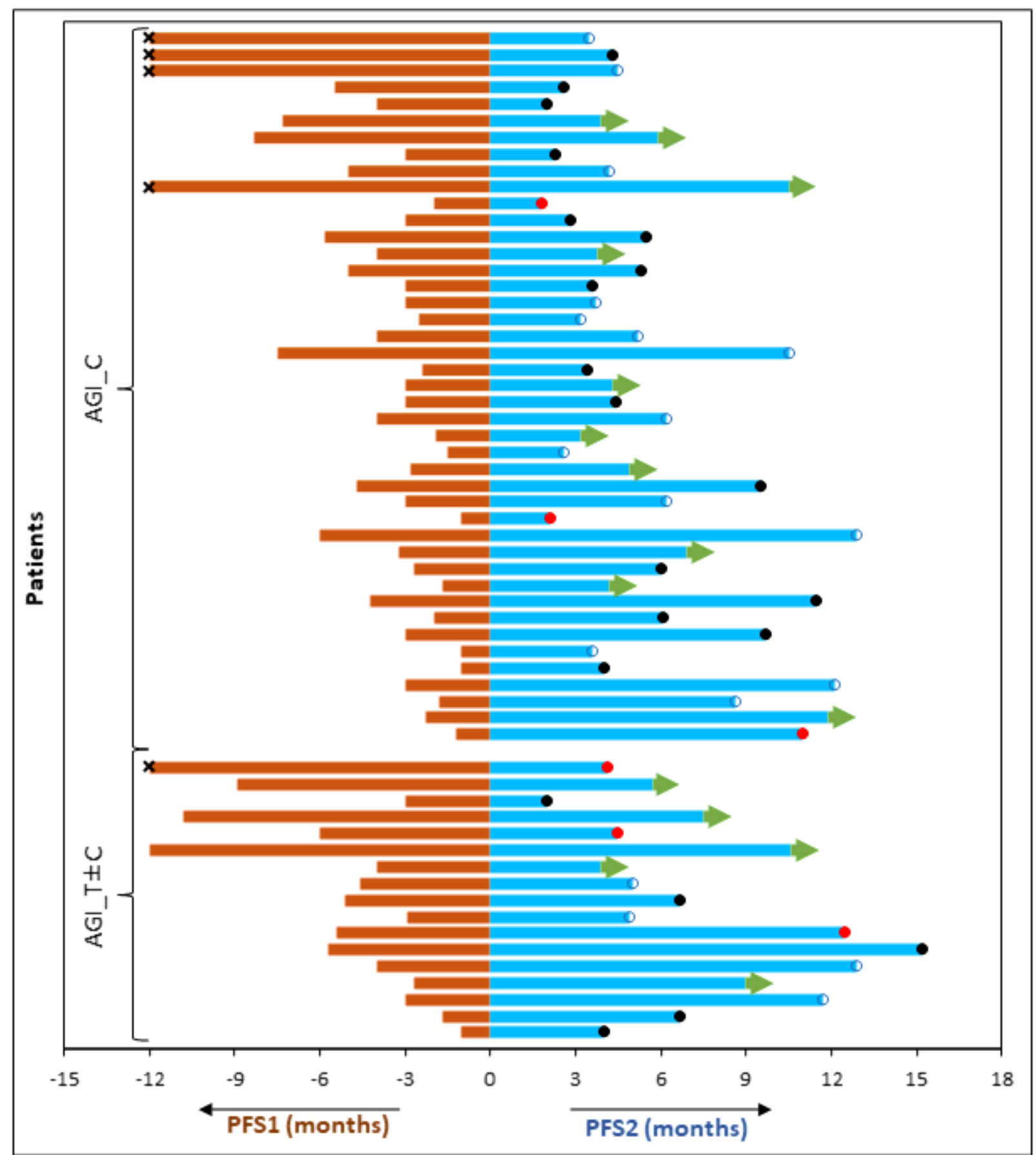

Figure 3

Comparison of Progression Free Survival on Last Failed Line of Systemic Treatment (PFS1) and on the Present Regimen (PFS2). PFS of each patient (months) on the last line of treatment (left) is compared with the PFS observed on ETA guided AGI combination therapy regimen (right). open circle: censored. red circle: demise; black circle: progression; green arrow: ongoing PFS; $x$ : PFS1 was $>12$ months. 


\section{Supplementary Files}

This is a list of supplementary files associated with this preprint. Click to download.

- 210321ETAAGISupp.xIsx

- 210321ETAAGISuppMethod.docx 